\title{
Situational Impairments to Mobile Interaction in Cold Environments
}

\author{
Zhanna Sarsenbayeva ${ }^{1}$, Jorge Goncalves ${ }^{1}$, Juan García ${ }^{1}$, Simon Klakegg ${ }^{1}$, \\ Sirkka Rissanen ${ }^{2}$, Hannu Rintamäki ${ }^{2}$, Jari Hannu ${ }^{3}$, Vassilis Kostakos ${ }^{1}$ \\ ${ }^{1}$ Center for Ubiquitous Computing, University of Oulu, Finland \\ ${ }^{2}$ Finnish Institute of Occupational Health, Oulu, Finland \\ ${ }^{3}$ Microelectronics Research Unit, University of Oulu, Finland \\ ${ }^{1}\left\{\right.$ firstname.lastname\}@ee.oulu.fi, ${ }^{2}\left\{\right.$ firstname.lastname\}@ttl.fi, ${ }^{3}\{$ firstname.lastname\}@oulu.fi
}

\begin{abstract}
We evaluate the situational impairments caused by cold ambient temperature on fine-motor movement and vigilance during mobile interaction. For this purpose, we tested two mobile phone applications that measure fine motor skills and vigilance in controlled temperature settings. Our results show that cold adversely affected participants' fine-motor skills performance, but not vigilance. Based on our results we highlight the importance of correcting measurements when investigating performance of cognitive tasks to take into account the physical element of the tasks. Finally, we identify a number of design recommendations from literature that can mitigate the adverse effect of cold ambiance on interaction with mobile devices.
\end{abstract}

\section{Author Keywords}

Smartphones; cold temperature; situational impairments; fine-motor movements; offset; vigilance; ubiquitous computing.

\section{ACM Classification Keywords}

H.5.m. Information interfaces and presentation (e.g., HCI): Miscellaneous.

\section{INTRODUCTION}

We investigate the effect of acute cold exposure on vigilance and fine-motor movements during mobile interaction. Despite the de-facto prominence of UbiComp experiments "beyond the desktop" and "in the wild" [26], relatively little work has investigated how interaction itself is shaped by non-conventional environments which can lead to situational impairments [47]. Situational impairment is a temporary loss of bodily function caused by a variety of factors including vibration, divided attention, diverted gaze, body motion, awkward postures, cold temperatures, clothing, rainwater, glare, uneven terrain, ambient noise, or encumbering baggage [15]. Specifically, very little work has systematically investigated how interaction with mobile devices is shaped by ambient temperature, even though ambient temperature substantially deviates geographically, seasonally, and diurnally.

There are two primary sets of human functions that govern how people interact with mobile devices: physical and cognitive. Physical aspects concern motor actions such as touching, pointing, and swiping. Cognitive aspects concern tasks such as finding and launching necessary applications, finding and activating commands, responding to output, and formulating language [37]. Our study focuses on how these two sets of functions are affected by ambient temperature, by considering performance in terms of fine-motor movements and vigilance respectively.

It is common in cold climates for users to interact with their devices in outdoor settings. Although exposure to cold in modern society is typically limited to short bursts, it remains substantial for industries such as agriculture, forestry, mining, factory and construction work, hunting, fishing, and military [27]. People working in these activity fields face prolonged exposure to cold conditions that can deteriorate their performance [46].

Since cooling of the tissue may result in degraded physical [38] and mental [31] performance, completing the same task in cold can require extra effort compared to a warm environment [27]. Physical interaction with mobile devices may also be adversely affected in cold due to muscular dexterity loss [22] and shivering, which may in turn disturb fine motor control. Hence, a better regulation of movements may be required to perform motor-tasks compared to a warm environment [38]. Further, research shows that discomfort caused by cold can adversely affect tasks requiring concentration and vigilance [27]. It also deflects attention from primary task and results in impaired cognitive performance $[42,43]$. Rich literature exists on how interaction with mobile devices is impaired by situational factors such as motion [15], divided attention [24], and ambient noise [47]. However, while it is well established that cold temperatures affect vigilance amongst other cognitive capabilities [42,43], and fine-motor movements [38], very little prior work has investigated the effect of cold temperatures have on mobile interaction.

Even though the effect of temperature has been reported extensively in literature, its impact on mobile device use has not been explored. This study is the first to do so, and our findings highlight the importance of adapting interfaces of mobile devices in cold settings, and the need to account for this effect when conducting experiments in cold temperatures. 


\section{RELATED WORK}

Situational impairments and mobile phone interaction

There is a lack of understanding of situational impairments and their impact on mobile interaction [47]. Goel et al. emphasise that situational impairments, such as dynamic state of the phone, might affect successful interaction with the device, because current mobile devices still lack appropriate awareness of environmental context [16]. Previous work has highlighted how situational impairments may worsen user's interaction with mobile device regardless of their abilities [23], since they can be caused by various factors, such as ambient temperature, ambient light [47], ambient noise [47] and movement [15]. Moreover, situational impairments might cause further accessibility issues for people with disabilities when attempting to use the device in one of the mentioned situations [23]. Also, a better understanding of situational impairments can be helpful in improving accessibility for those with more permanent physical impairments, since they both might affect users in a similar way [47]. Wobbrock argues that understanding situational impairments would increase our understanding of needs for improved accessibility and adaptive user interfaces [47].

Since cold-induced motor impairments and temporal disabilities may have common characterisation, it is important to consider literature on the use of touchscreen technology by motor-impaired individuals. Such individuals typically find conventional gestures challenging, often causing them to refrain from using touchscreen devices altogether [50]. Duff et al. [8] report that motor-impaired individuals make more mistakes than individuals without disabilities when hitting targets on a touchscreen. Further, Wobbrock et al. [48] showed that tremor and fatigue adversely affected user's ability to control their movements on PDA screens using stylus. Kane et al. summarise problems faced by people with motor and visual disabilities when using mobile phones such as difficult interfaces with small buttons and screens, causing difficulties while reading [24]. Therefore, design implications from this area of research can potentially be adapted for users without disabilities when suffering from cold-induced decreases in manual dexterity.

\section{Effect of cold on fine-motor and cognitive skills}

Previous work has highlighted that cold exposure can severely affect fine-motor skills [6,32]. Tasks involving manipulations of fingers are more adversely affected in cold than those involving hand and arm manipulations [44,49]. For instance, standardised Pegboard tests show that decreased manual dexterity significantly increases reaction time (i.e. target acquisition time) and lowers accuracy in manual tasks [22].

Further, Havenith et al. demonstrate that finger dexterity decreases sharply when the skin temperature drops below $15^{\circ} \mathrm{C}$ [19]. Moreover, the more finger dexterity the task requires, the bigger the performance loss will be. Even though this effect is well documented, very little prior work has directly studied the effects of cold temperature on users' interaction with technology. As one example, Blomkvist [3] used a target size in a Fitts' acquisition task [12] to investigate the effect of cold hands on desktop input using a mouse, two trackballs (small, large), and a Wacom tablet with two pens (thin, thick). The experiment consisted of four sessions, with one of the sessions performed with cold hands. In that session, participants' hands were immersed into a large bowl with a mixture of snow and water, and kept immersed until the finger skin temperature reached $11{ }^{\circ} \mathrm{C}$. The study reported that participants with cold hands performed slower when using trackballs which require higher finger dexterity, regardless the target size.

Regarding cognitive performance, previous work has shown that cold can have a significant impact due to the distracting power of a more stressful environment than usual [42,45]. For example, Daanen et al. found a deteriorating effect of cold on driving performance, and reported a $16 \%$ decrease in driving performance at temperatures of $5{ }^{\circ} \mathrm{C}$ compared to driving performance at 20 ${ }^{\circ} \mathrm{C}$ [7]. Vaughan and Strauss also documented considerable degradation in cognitive performance after exposure to cold water $\left(4.5^{\circ} \mathrm{C}\right)$ compared to exposure to warmer water $(15.5$ $\left.{ }^{\circ} \mathrm{C}\right)$. Accuracy for solving simple arithmetic tasks and navigation problems fell by $11 \%$ and $9 \%$ respectively. Vigilance performance decreased by $3 \%$ in target detection task alongside with detection time, which increased by $26 \%$ [45].

Pilcher et al. reported that below $10{ }^{\circ} \mathrm{C}$ cognitive skills such as reasoning, learning and memory are impaired [34]. Flouris et al. observed significant vigilance deterioration in a target hitting task in ambient temperatures of $-20{ }^{\circ} \mathrm{C}$ within 45 minutes of exposure to cold [13]. They also report more deteriorated vigilance in women in comparison to men. Hence, gender needs to be controlled in studies investigating physiological response to cold. Reaction time to complete a task is considered to be one of the indicators that measure vigilance [13]. Several studies report longer response times when cognitive tasks are performed while participants are exposed to cold air or water $[6,40]$. Furthermore, exposure to a cold room at $-5{ }^{\circ} \mathrm{C}$ increased error rate in 8 -choice reaction time tasks $[10,11]$.

We hypothesise that cold temperatures can severely affect smartphone usage due to decreased manual dexterity [38] and vigilance [45]. Particularly, we hypothesise that from a fine-motor performance perspective, offset and reaction time of touch will be larger in cold than in warm. From a cognitive skills perspective, we hypothesise that due to the adverse effect of cold on vigilance, users will take longer time and make more mistakes when asked to remember and find an icon, a common task in mobile phone use. 


\section{STUDY}

\section{Software}

Two custom Android applications called TapCircle and FindIcon were developed for this study. The TapCircle application was designed to quantify participants' finemotor performance in a target acquisition task, while the FindIcon application was used to quantify participants' vigilance in a search task.

The TapCircle application displayed circular targets of 135 pixels radius, randomly appearing on a 4x6 grid [20], one at a time. The grid positions were numbered as shown in Figure 1.

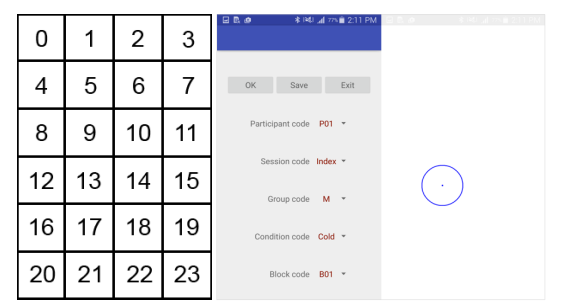

Figure 1. Grid with 24 positions and the interface of TapCircle application.

Every target had an indicated center and participants were instructed to tap the center of the target as precisely and as quickly as possible. We ensured that targets appeared in each of the 24 grid positions at least once in order to have data points on every grid position. Figure 1 shows the interface used by the researchers to set the conditions, and the application used by the participants. This application logged the position of the grid where a target was drawn, coordinates of the target's center and participant's touch, and elapsed time. Only taps inside the circle were subsequently retained during data analysis. We decided to use a custom target selection task instead of the standard reciprocal tapping task (ISO 9241-9), so as to more closely emulate a standard smartphone "Home Page" interface (circles were positioned in spots where application icons appear).

In the FindIcon application, participants had to locate and click on a particular target icon amongst a set of 24 icons in a $4 \times 6$ grid that emulated a typical home screen of a mobile phone. The target icon was first shown to participants before each trial, and participants could look at the target icon for as long as necessary until they memorised it. The application was designed so that in each condition each of the 24 icons would be designated as the target in a random order, and that every grid position would host a target icon in a random order. This randomisation minimised any possible learning effects. The set of 24 icons was chosen from the list of most downloaded applications with more than 100 million downloads each [25]. Figure 2 shows the interface used by the researchers to set the conditions, and the interface of the application seen and used by the participants. We recorded the time spent on memorising the target icon, the time taken to locate and click the target icon, the grid position where the target icon was shown (including $\mathrm{X}$ and $\mathrm{Y}$ coordinates of the start and center points of the icon), and the coordinates and timestamp of all touches made by participants. The application required participants to click on the correct icon before proceeding to the next trial, and recorded the number of wrong attempts before the correct icon was clicked.

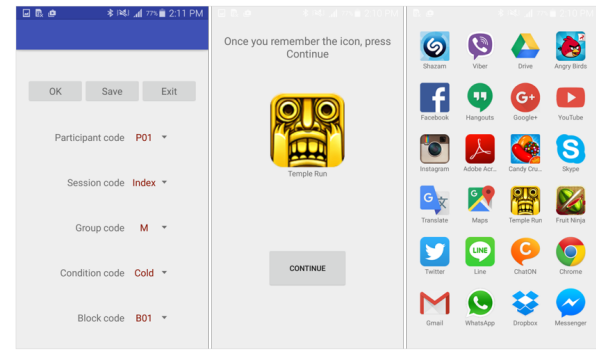

Figure 2. Interface of FindIcon application

Participants performed tasks on both of our applications, counterbalancing the order.

\section{Participants and Apparatus}

Participants were recruited through mailing lists and snowball recruitment. Twenty-four participants aged 18 to 35 years $(\mathrm{M}=24.64, \mathrm{SD}=4.55)$ enrolled (12 male, 12 female). Gender was balanced since literature suggests that it affects physiological response to cold $[2,13,36]$. We controlled for acclimatisation to cold temperature by ensuring that all participants had lived in cold climates (e.g., Scandinavia) for more than six months. Participants were required to have owned a smartphone for more than a year.

Participants' clothing was controlled by instructing them to wear a single layer of trousers, one pair of socks and top garment on the day of the study. During the experiment, every participant was asked to wear additional winter attire provided by us, consisting of a winter jacket and hat (Figure $3)$. The participants were asked to not wear gloves nor warm their hands through movement, rubbing, or the pockets. Each participant was paid 40 Euros for participating.

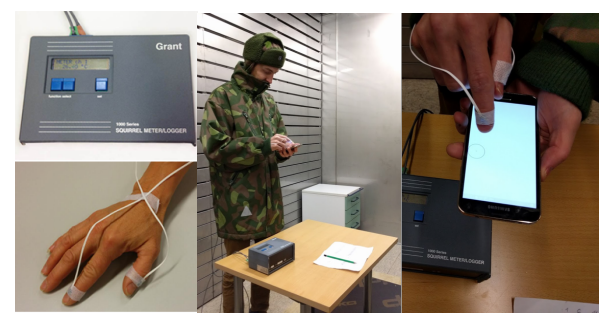

Figure 3. Grant Squirrel meter/logger (series 1000), a participant's hand with thermal sensors attached, and a cold chamber setup.

Participants used a Samsung Galaxy S5 smartphone running Google's Android 5.0 (Lollipop) operating system with a 5.1-inch screen sized 1080 by 1920 pixels. The experiment took place in two adjacent chambers hosted at 
an arctic medical facility. The warm room had a controlled ambient temperature of $20{ }^{\circ} \mathrm{C}$, wind velocity below $0.1 \mathrm{~m} / \mathrm{s}$, and humidity of $30-35 \%$. The cold chamber had a controlled ambient temperature of $-10{ }^{\circ} \mathrm{C}$, wind velocity below $0.1 \mathrm{~m} / \mathrm{s}$, and humidity of $70-75 \%$. Finger temperature was measured using two thermistors (Model 427, YSI Inc. USA) attached to the index finger and thumb of each participant's dominant hand, just below the nail. Thermal data was logged every 1 second using a mobile batterypowered Grant Squirrel meter/logger series 1000 (Figure 3). We collected this information to verify the temperature drop in the cold chamber as well as temperature rise in the warm room.

\section{Design and Procedure}

The study followed a within-subjects experimental design. The first independent variable was the experimental setting: warm room or cold room. The second independent variable was the finger used for target acquisition: index finger or thumb. This variable was coupled with holding posture, since thumb was used in one-handed operation while index finger requires two-handed operation. The experimental design was approved by the Human Sciences ethics committee of our university.

Participants arrived to the warm room, where they were initially briefed on the purpose of the study. We then recorded their personal details (age, gender, dominant hand) and asked them to sign a consent form if they agreed to study specifications. We then attached the two thermistors to their dominant thumb and index fingers while making sure the wires did not interfere with participants' movement. For training we asked participants to freely interact with our smartphone and both applications so they become accustomed to the setting, and subsequently to complete one full session with both of the interaction modes (one-handed and two-handed), which we discarded from our data analysis. Reception and training of participants lasted 20-30 minutes, and was designed to ensure that learning effects [19] and performance variations [35] are minimised, and participants' finger temperature is stabilised.

After training, participants were dressed in our winter attire and shown to the cold chamber to begin the experiment. Each participant completed 4 experimental sessions as follows: cold-warm-cold-warm (Figure 4), and during the whole experiment participants were standing. We decided not to counterbalance the order in which participants experience the warm and cold rooms for two reasons. First, all participants were inevitably exposed to room temperature before beginning our study, and counterbalancing would require exposing half of the participants to the cold chamber 3 times. This is crucial because we wanted to observe fluctuations in finger temperature: if participants started in the warm room, it would not be possible to measure fluctuations in the first trial. Second, following ethical recommendations we decided to minimize the time we expose our participants to the cold chamber, keeping it to 2 visits for each participant.

Finally, we precisely timed participants' exposure to cold by running trials at specific time periods instead of waiting until participants reached certain finger temperatures.

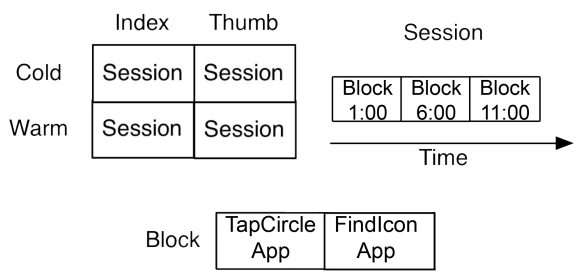

Figure 4. Experimental setup diagram

The 4 sessions tested either the thumb (one-handed) or index finger (two-handed) for interacting with the smartphone. The order of active finger was counterbalanced, and once a session was completed the participants switched rooms and continued with the next session. Thus, the order of the 4 sessions was either [coldthumb, warm-thumb, cold-index, warm-index] or [coldindex, warm-index, cold-thumb, warm-thumb]. In every session a researcher kept strict timing using a handheld timer. Once a participant entered a room, the scientist began the timer. When the timer reached 1:00 (1 minute), the participant was instructed to begin Block 1 in which both applications' task was completed. The order in which applications appeared was counterbalanced between participants. A block typically lasted around 2.5 minutes in TapCircle application and 1.5 minutes in FindIcon application, and once a block of tasks was finished for both applications, participants waited (with their hands lowered to a natural position) for the scientist to hand them back the phone and signal to begin the next block. The blocks were timed to begin at 1:00, 6:00, 11:00 within a given session. Hence, our experimental design controlled the exposure time of participants to the cold and warm rooms.

Once the experiment was completed, participants took part in a short interview session. Regarding both applications, we enquired participants about their subjective opinion if they were more precise and/or quick when completing the tasks in any of the conditions. We then asked them to identify parts of the screen that were in their opinion harder/easier to reach (in both applications) and find the icon (application FindIcon). We also asked participants to report which of the apps used in our experiment were also installed on their phones in order to determine if this affected time taken to find an icon. Finally, we enquired what strategy they used to find an icon, and whether shape, text or colour of the icons mattered.

\section{Results}

The experiment (including intake, training, and experiment) lasted about 90 minutes per participant, and the scientists observed 4 participants per day. Each participant completed 
a total of at least 288 correct circles and 288 correct icons hits. Overall, we collected 33,672 target hits from 24 participants for both applications $(26,448$ for TapCircle and 7,224 for FindIcon), and independent thermal data from 2 fingers per participant every 1 second. All data was timestamped to enable posthoc synchronisation. Further, data from left-handed participants $(\mathrm{N}=3)$ was mirrored in the $\mathrm{x}$-axis of screen position for consistency purposes.

We performed an initial analysis to test if there was a learning effect across the trial blocks. We found no significant improvements in performance as the experiment progressed over time. This was expected, since we provided extensive training at the start of our experiment and the tasks were simple by design.

\section{Smartphone Performance}

Our experiment used an off-the-shelf smartphone instead of a certified cold temperature device, to provide realism in our tests. We tested the smartphone's hardware performance in room temperature $\left(20{ }^{\circ} \mathrm{C}\right)$ and cold temperature $\left(-10^{\circ} \mathrm{C}\right)$ at identical timeframes as in our experiment (at 1 minute, 6 minutes, 11 minutes) using the AnTuTu benchmarking software, which reports a benchmark score that measures performance. Our results showed non-significant impact of cold temperature on the performance of the smartphone $(\mathrm{t}(2)=-2.21, \mathrm{p}=0.16)$.

\section{Task Performance in TapCircle Application}

We built a model that describes the effect of cold exposure on the time taken to tap a circle. We first ran a stepwise multiple regression analysis to predict the time taken to tap a circle. The final prediction model contained all 5 possible predictors (environmental condition, interaction mode, $\mathrm{x}$ and y center coordinates of target grid position, and gender) and was built in 1 step without any of the variables being removed. The model is statistically significant $(\mathrm{F}(5,26442)$ $=649, \mathrm{p}<0.01)$ and described approximately $11 \%$ of variance of time taken to tap a circle $\left(\mathrm{R}^{2}=0.11\right.$, Adjusted $\left.\mathrm{R}^{2}=0.11\right)$. All factors were significant (Table 1 ).

\begin{tabular}{lcccc} 
& Est. & Std. Err. & t & p \\
(Intercept) & $6.53 \mathrm{e}-01$ & $4.09 \mathrm{e}-03$ & 159.80 & $<2 \mathrm{e}-16$ \\
Condition (warm) & $-1.54 \mathrm{e}-02$ & $2.53 \mathrm{e}-03$ & -6.05 & $1.45 \mathrm{e}-09$ \\
Interaction mode (one hand) & $1.21 \mathrm{e}-01$ & $2.54 \mathrm{e}-03$ & 47.58 & $<2 \mathrm{e}-16$ \\
X center coordinate & $-7.04 \mathrm{e}-05$ & $4.18 \mathrm{e}-06$ & -16.83 & $<2 \mathrm{e}-16$ \\
Y center coordinate & $2.16 \mathrm{e}-05$ & $2.41 \mathrm{e}-06$ & 8.98 & $<2 \mathrm{e}-16$ \\
Gender (male) & $-5.73 \mathrm{e}-02$ & $2.53 \mathrm{e}-03$ & -22.61 & $<2 \mathrm{e}-16$ \\
\hline
\end{tabular}

Table 1. Coefficients of the model predicting the effect of cold exposure on the time taken to tap a circle.

Table 1 shows that participants took less time to tap the circles in the warm room than in the cold chamber, and took longer when interacting with the phone in one-handed mode compared to two-handed interaction mode. Male participants were significantly faster than female participants when completing the task of tapping circles. $\mathrm{X}$ and $\mathrm{Y}$ center coordinates of the circles were significant for the model; however, we should not interpret them in a linear fashion, as shown in Figure 5. In other words, greater $\mathrm{X}$ or $\mathrm{Y}$ coordinates do not necessarily mean better performance and vice-versa.

We next built a model that describes the effect of cold exposure on the mean offset when tapping a circle. The final prediction model contained 4 of the 5 predictors (environmental condition, interaction mode, $\mathrm{X}$ and $\mathrm{Y}$ center coordinates of grid position) and was obtained in 2 steps by removing one variable (gender). The model is statistically significant $(\mathrm{F}(4,26443)=103.4, \mathrm{p}<0.01)$ and explained $2 \%$ of variance of time taken to find an icon $\left(\mathrm{R}^{2}=0.02\right.$, Adjusted $\mathrm{R}^{2}=0.02$ ). All remaining factors were statistically significant as can be seen in Table 2 .

\begin{tabular}{lcccc} 
& Est. & Std. Err. & t & p \\
(Intercept) & 48.18 & 1.01 & 47.51 & $<2 \mathrm{e}-16$ \\
Condition (warm) & -1.93 & 0.66 & -2.93 & $<0.01$ \\
Interaction mode (one hand) & 5.63 & 0.66 & 8.51 & $<2 \mathrm{e}-16$ \\
X center coordinate & -0.02 & $<0.01$ & -16.36 & $<2 \mathrm{e}-16$ \\
Y center coordinate & 0.01 & $<0.01$ & 7.56 & $4.17 \mathrm{e}-16$ \\
\hline
\end{tabular}

Table 2. Coefficients of the model predicting the effect of cold exposure on the offset.

As shown in Table 2, the offset was smaller in the warm room (vs. the cold room). Further, the offset size was larger for one-handed (vs. two-handed). In addition, position of $\mathrm{X}$ and $\mathrm{Y}$ center coordinates of circles was significant in predicting the offset; however, as reported above, $\mathrm{X}$ and $\mathrm{Y}$ coordinates do not linearly relate to the offset size.

Mean offsets between target center and participant click are shown in Figures 5 and 6, with arrowheads indicating the mean direction and mean length of the offset vector. The colours of the circles also indicate the length of the offset, with lighter colour indicating longer offset and darker colour indicating shorter offset.

Table 3 shows the mean offset and time taken to tap a target in the TapCircle app. We estimated the effect size for offset between cold and warm environments for both two-handed (Cohen's $\mathrm{d}=0.04$ ) and one-handed interaction mode (Cohen's $d=0.04)$. We also estimated effect size for time taken to hit a target for two-handed (Cohen's $d=0.09$ ) and one-handed interaction modes (Cohen's $\mathrm{d}=0.07$ ).

\begin{tabular}{ccc}
\multicolumn{3}{c}{ Interaction mode } \\
Two-handed $($ Index) & One-handed (Thumb) \\
\multirow{2}{*}{ Cold } & Offset $=42.66(33.04) \mathrm{px}$ & Offset $=49.30(72.39) \mathrm{px}$ \\
& Time $=603(144.62) \mathrm{ms}$ & Time $=732(254.51) \mathrm{ms}$ \\
Warm & Offset $=41.34(40.89) \mathrm{px}$ & Offset $=46.47(58.22) \mathrm{px}$ \\
& Time $=593(137.89) \mathrm{ms}$ & Time $=709(248.36) \mathrm{ms}$ \\
\hline
\end{tabular}

Table 3. Mean offset and mean time taken to hit a target for TapCircle application. SD reported in brackets. 

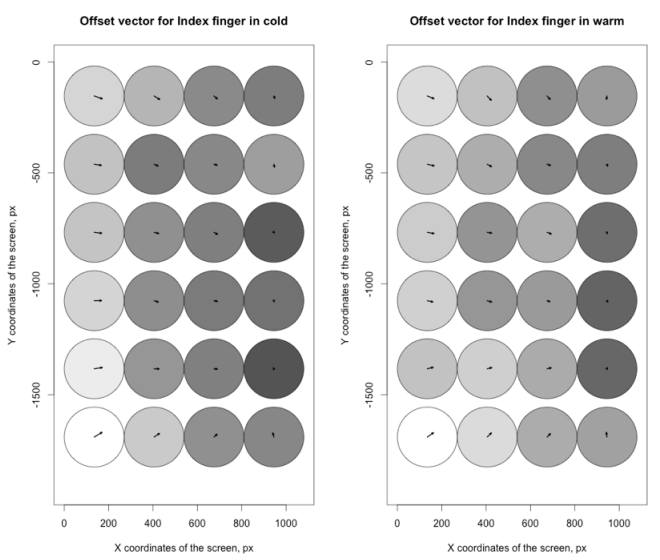

Figure 5. a) Offset vectors for index finger in cold, b) Offset vectors for index finger in warm
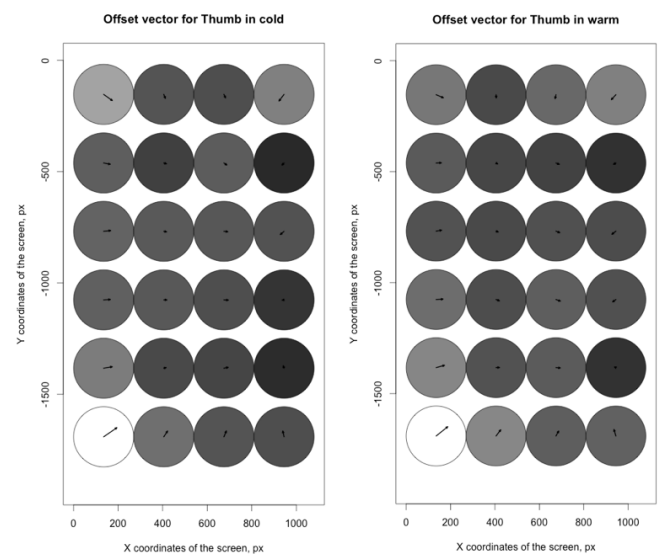

Figure 6. a) Offset vectors for thumb in cold, b) Offset vectors for thumb in warm

\section{Task Performance in Findlcon Application}

\section{Data Treatment}

We followed the recommendations from Flouris [13] and quantified vigilance using reaction time and error. In the FindIcon application, participants had to complete the task of finding and clicking a target icon, which consisted of three subtasks: 1) memorise the target icon, 2) locate the target icon amongst 24 icons, 3) click on the target icon. The time for subtask 1 was recorded separately by our software. However, the time for subtasks 2 \& 3 was inevitably aggregated and recorded as a single value.

To quantify vigilance, we were mostly interested in subtask 2: the time taken to search and locate a particular target icon. Hence, in our analysis we had to develop a way to measure subtask 2 and exclude the time taken to complete subtask 3 .

Due to our experimental design, the data from the TapCircles trials gave us an estimate of the average time needed for a participant to click an icon at a particular grid position, using either thumb or index finger, in either the warm or cold rooms. Essentially, this mean value is an estimate for the duration of subtask 3, and therefore we were able to subtract it from our data to arrive at an estimation for subtask 2 . We note that different values were calculated and subtracted for each participant, room (warm vs. cold), interaction mode (index vs. thumb), and block (minute 1:00 vs. minute 6:00 vs. minute 11:00).

Finally, we labeled each icon based on colour. Four researchers rated the icons according to the icons' dominant colours. This classification revealed a taxonomy with 8 categories of colours: blue, red, green, purple, yellow, brown, orange, and mixed (for icons with no dominant colour). An interrater reliability analysis using the Kappa statistic was performed to determine consistency among raters, which showed strong agreement between raters $(\kappa=$ $0.77, \mathrm{p}<0.01)$. Overall there were 7 blue icons ("Facebook", "Shazam", "Skype", "Dropbox", "Translate", "Messenger", "Twitter"), 6 mixed colour icons ("Drive", "Chrome", "Candy Crush Saga", "Angry birds", "Maps", "Fruit Ninja"), 4 red icons ("Adobe Acrobat reader", "YouTube", "Gmail", "Google+"), 3 green icons ("Line", "Whatsapp", "Hangouts"), and 1 of each: purple ("Viber"), yellow ("TempleRun"), brown ("Instagram"), orange ("ChatOn").

\section{Time to memorise}

We analysed if the time taken to memorise each target icon was affected by the environmental condition and interaction mode (Table 2). A two-way repeated measures ANOVA was run to test the effect of the environmental condition (cold or warm room) and interaction mode (one-handed or two-handed) on the time taken to memorise the icon. The results showed a significant effect for environmental condition $(\mathrm{F}(1,6878)=128.640, \mathrm{p}<0.01)$ but not interaction mode $(\mathrm{F}(1,6878)=1.179, \mathrm{p}<0.29)$, and a significant interaction effect $(\mathrm{F}(1,6878)=278.525, \mathrm{p}<$ 0.01 ). In the cold chamber participants were significantly slower in memorising an icon than in the warm room across both one-handed and two-handed interaction modes. Finally, we estimated the effect size for time taken to memorise an icon between cold and warm environments, separately for one-handed interaction (Cohen's $d=0.23$ ) and two-handed interaction (Cohen's d $=0.22$ ).

\section{Modeling the impact of cold exposure}

We built a model that describes the effect of cold on the time taken to find and click the target icon. We first ran a stepwise multiple regression analysis to predict the total time taken to locate and click a target icon. The final prediction model contained 5 of the 6 possible predictors (environmental condition, interaction mode, $\mathrm{X}$ and $\mathrm{Y}$ center coordinates of target grid position, and icon colour) and was built in 2 steps by removing 1 variable (gender). The model is statistically significant $(F(11,6248)=33.30, p<0.01)$ and describes approximately $5.5 \%$ of the variance of total time taken to search and locate a particular target icon $\left(\mathrm{R}^{2}=\right.$ 0.055 , Adjusted $R^{2}=0.054$ ). All factors were statistically significant and are presented in Table 4. 


\begin{tabular}{lccc|c} 
& Est. & Std. Err. & t & p \\
(Intercept) & $1.60 \mathrm{e}+00$ & $3.23 \mathrm{e}-02$ & 49.48 & $<2 \mathrm{e}-16$ \\
Condition (warm) & $-4.06 \mathrm{e}-02$ & $1.89 \mathrm{e}-02$ & -2.15 & 0.03 \\
Interaction mode (one hand) & $1.31 \mathrm{e}-01$ & $1.89 \mathrm{e}-02$ & 6.93 & $4.64 \mathrm{e}-12$ \\
X center coordinate & $-2.63 \mathrm{e}-04$ & $3.32 \mathrm{e}-05$ & -7.93 & $2.65 \mathrm{e}-15$ \\
Y center coordinate & $1.31 \mathrm{e}-04$ & $1.92 \mathrm{e}-05$ & 6.80 & $1.11 \mathrm{e}-11$ \\
Icon colour (brown) & $-1.84 \mathrm{e}-01$ & $4.97 \mathrm{e}-02$ & -3.70 & $<0.01$ \\
Icon colour (green) & $-2.52 \mathrm{e}-01$ & $3.21 \mathrm{e}-02$ & -7.84 & $5.40 \mathrm{e}-15$ \\
Icon colour (mix) & $4.64 \mathrm{e}-02$ & $2.58 \mathrm{e}-02$ & 1.80 & 0.07 \\
Icon colour (orange) & $-3.87 \mathrm{e}-01$ & $4.92 \mathrm{e}-02$ & -7.87 & $4.27 \mathrm{e}-15$ \\
Icon colour (purple) & $-1.27 \mathrm{e}-01$ & $4.93 \mathrm{e}-02$ & -2.58 & $<0.01$ \\
Icon colour (red) & $-2.25 \mathrm{e}-01$ & $2.90 \mathrm{e}-02$ & -7.75 & $<1.06 \mathrm{e}-14$ \\
Icon colour (yellow) & $-2.34 \mathrm{e}-01$ & $4.93 \mathrm{e}-02$ & -4.81 & $1.53 \mathrm{e}-06$ \\
\hline
\end{tabular}

Table 4. Coefficients of the model predicting the effect of cold exposure on the time taken to find+tap the target icon.

Table 3 shows that in the warm room participants required less time to find and tap the target icon (vs. cold). In onehanded interaction mode participants took longer to complete the task (vs. two-handed). Blue icons took significantly longer time to be found and clicked in comparison to brown, green, orange, purple, red, and yellow icons.

We next built a model that describes the effect of cold on the time taken to find the target icon, i.e. adjusting time to exclude the time needed to click the target icon. The final prediction model contained 4 of the 6 predictors ( $\mathrm{x}$ and $\mathrm{y}$ center coordinates of grid position, icon colour, and gender) and was achieved in 3 steps by removing two variables (environmental condition and interaction mode). This removal was expected, as time was corrected to exclude the physical component of clicking the icon. The model is statistically significant $(\mathrm{F}(10,6249)=28.93, \mathrm{p}<0.01)$ and explained $4.4 \%$ of variance of time taken to find an icon $\left(\mathrm{R}^{2}\right.$ $=0.044$, Adjusted $\mathrm{R}^{2}=0.043$ ). All of the factors within the model are statistically significant as shown in Table 5. We estimated the effect size for time taken to find an icon between cold and warm environments for one-handed interaction mode (Cohen's $\mathrm{d}=0.03$ ) and two-handed mode (Cohen's $d=0.06)$.

Blue icons took significantly longer time to be found. Moreover, male participants took significantly less time than females. This corresponds with previous results in literature [13], which reported more deteriorated vigilance in women when compared to men.

\begin{tabular}{lcccc} 
& Estimate & Std. Err. & t & p \\
(Intercept) & $9.53 \mathrm{e}-01$ & $3.07 \mathrm{e}-02$ & 31.04 & $<2 \mathrm{e}-16$ \\
X center coordinate & $-2.05 \mathrm{e}-04$ & $3.26 \mathrm{e}-05$ & -6.29 & $3.35 \mathrm{e}-10$ \\
Y center coordinate & $-1.10 \mathrm{e}-04$ & $1.89 \mathrm{e}-05$ & 5.82 & $6.12 \mathrm{e}-09$ \\
Icon colour (brown) & $-1.83 \mathrm{e}-01$ & $4.89 \mathrm{e}-02$ & -3.74 & $<0.01$ \\
Icon colour (green) & $-2.55 \mathrm{e}-01$ & $3.16 \mathrm{e}-02$ & -8.08 & $7.86 \mathrm{e}-16$ \\
Icon colour (mix) & $3.97 \mathrm{e}-02$ & $2.53 \mathrm{e}-02$ & 1.57 & 0.12 \\
Icon colour (orange) & $-3.79 \mathrm{e}-01$ & $4.84 \mathrm{e}-02$ & -7.82 & $6.16 \mathrm{e}-15$ \\
Icon colour (purple) & $-1.36 \mathrm{e}-01$ & $4.85 \mathrm{e}-02$ & -2.81 & $<0.01$ \\
Icon colour (red) & $-2.32 \mathrm{e}-01$ & $2.85 \mathrm{e}-02$ & -8.14 & $4.93 \mathrm{e}-16$ \\
Icon colour (yellow) & $-2.51 \mathrm{e}-01$ & $4.85 \mathrm{e}-02$ & -5.18 & $2.32 \mathrm{e}-07$ \\
Gender (male) & $4.05 \mathrm{e}-02$ & $1.87 \mathrm{e}-02$ & 2.17 & 0.03 \\
\hline
\end{tabular}

Table 5. Coefficients of the model predicting the effect of cold exposure on the time taken to find the target icon.
Contrasting the two models indicates that when only cognitive effort is considered, the environmental condition and interaction mode become negligible factors in predicting time taken to find an icon. Ergo, environmental condition and interaction mode significantly affect physical aspects of interaction. Table 6 presents average values of time taken to find an icon for each condition.

\begin{tabular}{|c|c|c|}
\hline & \multicolumn{2}{|c|}{ Interaction mode } \\
\hline & Two-handed (Index) & One-handed (Thumb) \\
\hline \multirow{2}{*}{ Cold } & $\begin{array}{l}\text { Time to memorise }=854 \\
(196.47) \mathrm{ms}\end{array}$ & $\begin{array}{l}\text { Time to memorise }=922 \\
(229.52) \mathrm{ms}\end{array}$ \\
\hline & $\begin{array}{l}\text { Time to find }=873(727.11) \\
\mathrm{ms}\end{array}$ & $\begin{array}{l}\text { Time to find }=889(925.83) \\
\mathrm{ms}\end{array}$ \\
\hline \multirow{2}{*}{ Warm } & $\begin{array}{l}\text { Time to memorise }=815 \\
(150.15) \mathrm{ms}\end{array}$ & $\begin{array}{l}\text { Time to memorise }=875 \\
(185.05) \mathrm{ms}\end{array}$ \\
\hline & $\begin{array}{l}\text { Time to find }=834(626.37) \\
\mathrm{ms}\end{array}$ & $\begin{array}{l}\text { Time to find }=867(871.16) \\
\mathrm{ms}\end{array}$ \\
\hline
\end{tabular}

Table 6. Mean time to memorise a target icon and find a target icon for FindIcon application. SD reported in brackets.

Figures 7 and 8 show the difference in time taken to find an icon for each grid position in both interaction modes and environmental condition.

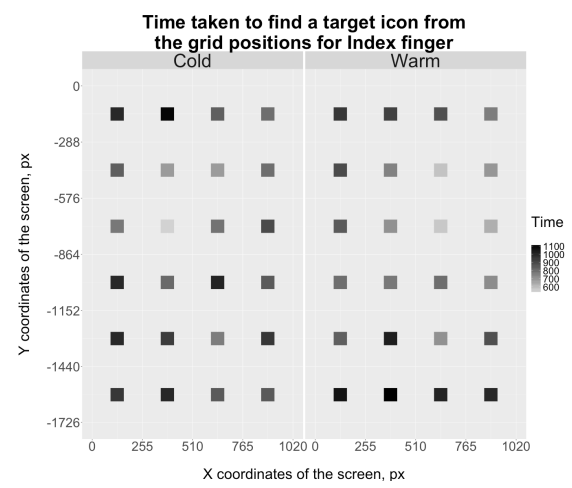

Figure 7. a) Time taken to find a target icon on the grid position for index finger in cold, b) Time taken to find a target icon on the grid position for index finger in warm

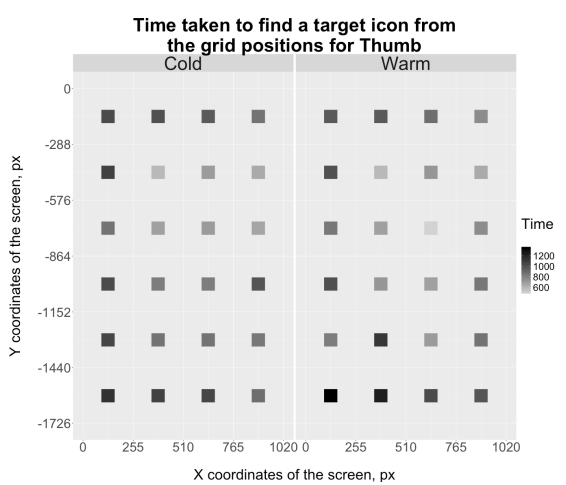

Figure 8. a) Time taken to find a target icon on the grid position for thumb in cold, b) Time taken to find a target icon on the grid position for thumb in warm 


\section{Error}

A Chi-square test was conducted to analyse the relationship between environmental condition and if an error was made when trying to finding an icon, and no significant relationship was found $\left(\chi^{2}(1,1)=0.12, \mathrm{p}=0.73\right)$. Similarly, no significant relationship was found between interaction mode and if an error was made when trying to finding an icon $\left(\chi^{2}(1,1)<0.01, \mathrm{p}=0.97\right)$. However, a Chi-square test showed a significant effect of icon colour on if an error was made when trying to find an icon $\left(\chi^{2}(1,7)=26.32\right.$, $\mathrm{p}<$ $0.01)$. Participants made more errors when finding blue icons compared to orange and purple. Finally, no significant relationship was found between participants that had installed the application of the target icon they were looking for and if an error was made when trying to find an icon $\left(\chi^{2}(1,7)<0.01, \mathrm{p}=0.96\right)$.

\section{Interview results}

During our interviews the majority of participants (16 out of 24) felt they were less precise in cold rather than in warm, while performing the tasks on TapCircle application. When asked for the reason, sense of cold and numb fingers were the main ones named: "I was less precise in cold, because my fingers were numb" (P14), "The precision was slightly worse in the cold due to frozen fingers" (P20), "My peak physical performance is more adjusted to warm" (P09).

Interestingly enough 6 out of 24 participants thought they were equally precise in both cold and warm conditions. Moreover, two participants thought they were more precise in the cold than in the warm, "because I wanted to get the task done, hence focused on pressing the circles" (P24). Overall, this subjective opinion is inline with our findings that showed that in the cold they were less precise than in the warm. When asked about particular positions on the screen that took longer time to access in a one-handed interaction mode (thumb finger), most participants agreed that corners of the screen along with the left side edge of the screen were the most difficult ones to access, hence required more time. Again, their answers correspond with our quantitative findings, indicating the top left and bottom left corners as the slowest to press.

As for the FindIcon application, when asked what strategy they used to find the target icon, all but one participants answered that the colour was the major aspect. Moreover, some participants elaborated on the colour aspect of the icons by saying "Fruit Ninja and Candy Crush icons were more difficult to find because they were colourful" (P17) or "their colour was too messy" (P11). Several participants mentioned that blue icons were harder to find, since there was a high number of them, however 7 people claimed that the Facebook icon (blue) was very quick to find, 3 people claimed Shazam and Skype icons were quicker to find "because of the colour contrast" (P01, P20, P22). Our quantitative findings also showed significant higher time taken to find blue and mixed coloured icons.
Furthermore, when asked to elaborate on their search strategy, 10 participants said that they scanned the screen as a whole, 7 participants preferred scanning the center first, and 4 scanned top, then bottom. One of the participants noted that "When the icon was in the middle, I could directly see it" (P08), even though his strategy was to scan icon by icon starting from the top of the screen. The remaining participants reported not having a preferred search strategy. When asked to mark on the grid where it was quicker to find an icon, participants were consistent in selecting the center of the grid. These results correspond to our findings, which showed that corners of the grid (positions 0 and 20 especially) as well as the top and bottom parts of the grid took longer time to access and find an icon.

\section{DISCUSSION}

A decade ago Wobbrock described four trends in society and technology that have direct consequences for mobile interaction, one being the increasing amount of personal computing used away from the desktop [47]. This is because the context of mobile device use can vary substantially more than desktop computers [47]. Hence, dealing with situational impairments [39] of mobile use is an important, but often neglected research challenge. In this paper, we explore one example of a situational impairment, mobile interaction in cold environments.

\section{Fine-motor performance with mobile phones in cold environments}

An extensive body of work highlights the effects of exposure to cold temperatures on humans' fine-motor skills (e.g., $[22,27,38])$. Our findings show that participants were more precise in a TapCircle task in the warm room than the cold chamber. Previous work has showed that since users cannot feel the position of touchscreen keys or buttons, offsets for top left corner naturally shift towards the lowerright corner, and offsets for lower-right corner naturally shift towards the top-left corner [20]. Our results correspond with these findings and show that the offset skew is observed in both interaction modes, a factor that was not investigate in previous work [20].

Our results also demonstrate for the first time that the magnitude of the offset is larger in cold conditions, particularly in one-handed interaction mode. While the differences in offset may look relatively small between the two environmental exposure conditions (Table 1), these are likely to be exacerbated in repeated everyday phone use in cold environments, especially when we consider that target acquisition is a task that users repeatedly perform to interact with touch-screen devices.

In terms of time taken, participants' performance also dropped in the cold chamber. Again, the differences in onehanded interaction mode were more pronounced in the cold chamber. Taking into account the simplicity of the task, we argue that a more complex task, such as text entry or object manipulation, would lead to even higher differences 
between these two environmental exposure conditions. Moreover, several participants were not aware of their precision loss during their interaction with the mobile device, since in the interview sessions $25 \%$ of participants (6 out of 24) claimed they were equally precise in both cold and warm rooms. This example highlights the importance of passively identifying potential situational impairments so that the device can react independently of users' direct feedback.

Further, we highlight the effect of grid position on the time taken to tap a circle. Our findings agree with previous work by Park \& Han [33], but extends to both modes of interaction, since Park \& Han primarily considered onehanded thumb interaction. According to our findings, the corners of the screen and the upper \& lower edges of the screen are areas where it was harder to tap a circle. Given that in cold settings users' fine-motor skills deteriorate, these challenging areas of the screen become even harder to reach/press.

Our results provide an empirical basis to design devices and interfaces that adjust to better accommodate situational impairments induced by cold exposure [47]. This can be achieved on mobile phones by increasing the size of buttons in problematic areas of the screen, or activating accuracyimproving input techniques such as Fat Thumb [4] or GraspZoom [30]. Further, the smartphone interface may adapt in real-time to minimise the time users expose their hands to cold temperature. These changes can rely on both contextual [17] or sensor [16] information (e.g., location, ambient temperature, smartphone battery temperature) and users' behaviour. Another potential application of our findings is notifying the user of possible frostbite risk when their performance decreases when using a smartphone in cold temperatures.

\section{Cognitive performance with mobile phones in cold environments}

Unlike physical performance, deterioration of cognitive skills tends to occur after a longer time of exposure to cold. For example, Flouris et al. recorded adverse deterioration of vigilance after 45 minutes of exposure to cold in $-20{ }^{\circ} \mathrm{C}$, and was highly correlated to core body temperature [13]. In addition, several studies state that cognitive impairment happens when the core body temperature drops by $2-4{ }^{\circ} \mathrm{C}$, which can requires longer exposure to cold [6,14]. Hence, it is natural that shorter exposure to less extreme ambient temperatures may not have any significant effect on vigilance. Our study did not control for core temperature as this would require invasive methods of measurements and would therefore not represent realistic use conditions. However, it is expected that at a certain threshold cognitive impairment would affect users' input performance.

The effect of cold on recall and recognition is disputable, since deterioration in recall but not recognition was observed in some studies [1], contrary to other studies where decline in recognition but not recall was reported
[11]. Our findings demonstrate that in the cold room participants took significantly longer time to memorise an icon compared to the warm room, however the following subtasks were not significantly affected by the cold environment. Neither time, nor frequency of errors were significantly affected by cold exposure, and our results correspond to the results by Flouris et al. [13]. Another possible explanation beyond insufficient exposure time to cold may have been that the task was too simple to measure and record a significant drop in vigilance. However, it is also unlikely that in cold users would perform complex tasks on their mobile devices. We also avoided using text entry as a task due to the difficulty in distinguishing the timing for its cognitive and physical components.

Nonetheless, our results demonstrate a significant effect of grid position on the time taken to find an icon. The screen's corners and upper/lower edges require more time to find an icon, in comparison to central positions. This finding suggests that future work can consider how people search mobile screens, ideally with the use of an eye tracker. While prototypes that detect eye motion when using mobile phones exist $[5,29]$, to the best of our knowledge, no prior work has investigated favourable mobile screen areas for the human eye in this manner. Such work could also help to understand mobile interface design implications for users with visual impairments, if prioritised areas of user gaze were identified.

Similarly, our results show a significant effect of icon colour on time taken to find an icon and frequency of errors. Interestingly, icons with the most frequent colour (blue) and mixed colours took longer time to find, while blue icons were also more prone to induce errors. As colour is a fundamental aspect of human perception [28], it is important to mitigate its adverse effect on cognition, especially under high cognitive load. Most existing studies focus on two or three primary colours $[9,28,41]$, whereas in our study we had 8 colour groups. Our results suggest that the choice of colour can have a much more pronounced effect than ambient temperature. Crucially, this means that poor interface colour choices can be worse than freezing temperatures, at the levels explored in this paper, in terms of users' cognitive performance during mobile interaction.

Finally, the comparison of the two predictive models we have presented indicates the significance of environmental condition on physical impairment, but not cognitive. Due to the time correction in the second model, the physical factors were no longer significant (environmental condition and interaction mode). This is an important finding of our study, because it implies that designers should prioritise adjusting mobile interface designs in cold environmental conditions to improve physical interaction with mobile devices. Further, it shows the importance of accounting for physical factors when conducting experiments aimed at assessing cognitive performance. 


\section{Limitations}

This study had several limitations. We constrained the experimental task either by involving the index finger or thumb, unlike in naturalistic settings where users involve more fingers or interchange them while accomplishing a goal on their mobile device. However, these were the requirements to control and detect differences in the performance of our participants in the two main modes of operation. Further, we did not counterbalance the presentation of the cold and warm rooms due to our experimental design and ethical concerns, which may have had an effect on our results. Another limitation was that we did not control icons' colour, i.e. we did not have equal number of icons for each colour. However, controlling for colour would not allow us to simulate realistic home screen of a mobile device, since it is unlikely for a standard mobile device user to have equal number of icons for each colour. One more limitation was that some participants might have been more acclimated to cold climate conditions, for example were born in Nordic countries. Moreover, other factors such as metabolic rate affected cooling-down rates. Nevertheless, through our within-subjects design we mitigate the effect of these factors to provide a fair account of changes in performance due to exposure to cold temperatures.

Finally, we used a cold chamber to simulate cold climate conditions and did not run the study under the natural environmental settings. This allowed us to create fair conditions for running the experiment by maintaining constant temperature and controlling climate factors such as precipitations, wind chill, wind speed and humidity. Previous work by Blomkvist [3] used local immersion of a hand in snow-water mixture to cool participants' hands, but as a drawback cooling is induced locally and abruptly unlike in naturalistic settings.

\section{CONCLUSION \& FUTURE WORK}

Our work investigates the effect of cold temperature exposure on mobile interaction in one-handed (using thumb) and two-handed (using index finger) interaction modes with bare hands. We find that at the levels of cold exposure we used in our experiment, fine-motor movements are significantly affected during mobile interaction, however the effect on cognitive performance is not significant. Specifically, in a cold environment the touch accuracy decreases, the target acquisition offset is significantly longer across both interaction modes, and participants take significantly longer time to hit a target. Further, cognitive skills measured by time taken to find a target icon and error frequency are not significantly affected by the environmental condition, but are substantially affected by the colour of an icon and the location on the 4x6 screen grid. We also highlight that when investigating performance of cognitive tasks on mobile devices it is important to correct time measurements to account for finemotor movement and dexterity.
Our findings highlight the need for mobile interfaces to adapt for usage in cold settings, especially considering finemotor skills. For example, current smartphone input techniques for users with disabilities can be extended to cold environment scenarios. Future research is needed to investigate and identify not only the effect of cold but other situational impairments that can cause fine-motor and cognitive deterioration when using mobile technologies. Further research is also warranted to explore the effect of cold temperature on user performance when interacting with touchscreens of other technologies that are used outdoors, such as public displays where voice controls have been proposed as a potential solution [18,21].

\section{ACKNOWLEDGEMENTS}

This work is partially funded by the Academy of Finland (Grants 276786-AWARE，285062-iCYCLE， 286386CPDSS, 285459-iSCIENCE), and the European Commission (Grants PCIG11-GA-2012-322138, 645706GRAGE, and 6AIKA-A71143-AKAI).

\section{REFERENCES}

1. Alan D. Baddeley, William J. Cuccaro, Glen H. Egstrom, Gershon Weltman and Michael A. Willis. 1975. Cognitive Efficiency of Divers Working in Cold Water. Human Factors: The Journal of the Human Factors and Ergonomics Society 17, 5: 446-454.

2. M. L. Bartelink, A De Wit, H Wollersheim, A Theeuwes and T Thien. 1993. Skin vascular reactivity in healthy subjects: influence of hormonal status. $J$ Appl Physiol (1985) 74, 2: 727-32.

3. A. C. Blomkvist and G. Gard. 2000. Computer usage with cold hands; an experiment with pointing devices. Int J Occup Saf Ergon 6, 4: 429-50.

4. Sebastian Boring, David Ledo, Xiang Chen, Nicolai Marquardt, Anthony Tang and Saul Greenberg. 2012. The Fat Thumb: Using the Thumb's Contact Size for Single-handed Mobile Interaction. In Proceedings of the 14th International Conference on Human-computer Interaction with Mobile Devices and Services, ACM, 39-48. http://doi.acm.org/10.1145/2371574.2371582

5. Andreas Bulling and Hans Gellersen. 2010. Toward Mobile Eye-Based Human-Computer Interaction. IEEE Pervasive Computing 9, 4: 8-12. http://dx.doi.org/10.1109/MPRV.2010.86

6. S. R. K. Coleshaw, R. N. M. van Someren, A. H. Wolff, H. M. Davis and W. R. Keatinge. 1984. Impaired Memory Registration and Speed of Reasoning Caused by Low Body Temperature. Survey of Anesthesiology 28, 4: 340 .

7. Hein A. Daanen, Evert van de Vliert and Xu Huang. 2003. Driving performance in cold, warm, and thermoneutral environments. Applied Ergonomics 34, 6: 597-602.

8. Sacha N. Duff, Curt B. Irwin, Jennifer L. Skye, Mary E. Sesto and Douglas A. Wiegmann. 2010. The effect 
of disability and approach on touch screen performance during a number entry task. In Proceedings of the Human Factors and Ergonomics Society Annual Meeting, 54, 6, 566-570.

9. Andrew J. Elliot, Markus A. Maier, Arlen C. Moller, Ron Friedman and Jörg Meinhardt. 2007. Color and psychological functioning: The effect of red on performance attainment. Journal of Experimental Psychology: General 136, 1: 154.

10. Hadyn D. Ellis. 1982. The Effects of Cold on the Performance of Serial Choice Reaction Time and Various Discrete Tasks. Human Factors: The Journal of the Human Factors and Ergonomics Society 24, 5: 589-598.

11. Hadyn D. Ellis, Sylvia E. Wilcock and S. A. Zaman. 1985. Cold and performance: The effects of information load, analgesics and the rate of cooling. Aviation, Space, and Environmental Medicine.

12. P. M. Fitts. 1954. The information capacity of the human motor system in controlling the amplitude of movement. J Exp Psychol 47, 6: 381-91. http://dx.doi.org/10.1037/h0055392

13. Andreas D. Flouris, David A. Westwood and Stephen S. Cheung. 2007. Thermal Balance Effects on Vigilance During 2-Hour Exposures To $-20^{\circ} \mathrm{C}$. Aviation, Space, and Environmental Medicine 78, 7: 673-679.

14. Gordon Giesbrecht, J. L. Arnett, E Vela and G. K. Bristow. 1993. Effect of task complexity on mental performance during immersion hypothermia. Aviation, Space, and Environmental Medicine.

15. Mayank Goel, Leah Findlater and Jacob Wobbrock. 2012. WalkType: using accelerometer data to accomodate situational impairments in mobile touch screen text entry. In Proceedings of the SIGCHI Conference on Human Factors in Computing Systems, 2687-2696.

http://dx.doi.org/10.1145/2207676.2208662

16. Mayank Goel, Jacob Wobbrock and Shwetak Patel. 2012. GripSense: Using Built-in Sensors to Detect Hand Posture and Pressure on Commodity Mobile Phones. In Proceedings of the 25th Annual ACM Symposium on User Interface Software and Technology, ACM, 545-554. http://doi.acm.org/10.1145/2380116.2380184

17. Jorge Goncalves, Vassilis Kostakos, Simo Hosio, Evangelos Karapanos, and Olga Lyra. 2013. IncluCity: using contextual cues to raise awareness on environmental accessibility. In Proceedings of the 15th International ACM SIGACCESS Conference on Computers and Accessibility (ASSETS '13). Article 17 http://dx.doi.org/10.1145/2513383.2517030
18. Jorge Goncalves, Simo Hosio, Yong Liu, Y. and Vassilis Kostakos. 2014. Eliciting situated feedback: A comparison of paper, web forms and public displays. Displays 35, 1: 27-37.

19. G. Havenith, R. Heus and H. A. Daanen. 1995. The hand in the cold, performance and risk. Arctic Med Res 54 Suppl 2: 37-47.

20. Niels Henze, Enrico Rukzio and Susanne Boll. 2011. 100,000,000 Taps: Analysis and Improvement of Touch Performance in the Large. In Proceedings of the 13th International Conference on Human Computer Interaction with Mobile Devices and Services, ACM, 133-142. http://doi.acm.org/10.1145/2037373.2037395

21. Simo Hosio, Jorge Goncalves, Vassilis Kostakos, and Jukka Riekki. 2015. Crowdsourcing Public Opinion Using Urban Pervasive Technologies: Lessons From Real-Life Experiments in Oulu. Policy \& Internet 7, 2: 203-222.

22. Zhao X. Jin, Tom Plocher and Liana Kiff. 2007. Touch screen user interfaces for older adults: button size and spacing. Springer. http://dx.doi.org/10.1007/978-3540-73279-2 104

23. Shaun K. Kane. 2009. Context-enhanced Interaction Techniques for More Accessible Mobile Phones. SIGACCESS Access. Comput. 93: 39-43. http://dx.doi.org/10.1145/1531930.1531936

24. Shaun K. Kane, Chandrika Jayant, Jacob O. Wobbrock and Richard E. Ladner. 2009. Freedom to roam: a study of mobile device adoption and accessibility for people with visual and motor disabilities. In Proceedings of the 11th international ACM SIGACCESS conference on Computers and accessibility, 115-122.

25. List of Most downloaded Android applications. Retrieved 28/03/2016 from https://en.wikipedia.org/wiki/List_of_most_downloade d_Android_applications

26. Yong Liu, Jorge Goncalves, Denzil Ferreira, Simo Hosio and Vassilis Kostakos. 2014. Identity crisis of ubicomp?: mapping 15 years of the field's development and paradigm change. In Proceedings of the 2014 ACM International Joint Conference on Pervasive and Ubiquitous Computing (UbiComp '14), 75-86. http://dx.doi.org/10.1145/2632048.2632086

27. T. Makinen, L. Palinkas, D. Reeves, T. Paakkonen, H. Rintamaki, J. Leppaluoto and J. Hassi. 2006. Effect of repeated exposures to cold on cognitive performance in humans. Physiology \& Behavior 87, 1: 166-176.

28. Ravi Mehta and Rui Zhu. 2009. Blue or Red? Exploring the Effect of Color on Cognitive Task Performances. Science 323, 5918: 1226-1229. http://dx.doi.org/10.1126/science.1169144 
29. Emiliano Miluzzo, Tianyu Wang and Andrew T. Campbell. 2010. EyePhone: Activating Mobile Phones with Your Eyes. In Proceedings of the Second ACM SIGCOMM Workshop on Networking, Systems, and Applications on Mobile Handhelds, ACM, 15-20. http://doi.acm.org/10.1145/1851322.1851328

30. Takashi Miyaki and Jun Rekimoto. 2009. GraspZoom: Zooming and Scrolling Control Model for Singlehanded Mobile Interaction. In Proceedings of the 11th International Conference on Human-Computer Interaction with Mobile Devices and Services, ACM, 11:1-11:4. http://doi.acm.org/10.1145/1613858.1613872

31. L. Palinkas. 2001. Circannual pattern of hypothalamicpituitary-thyroid (HPT) function and mood during extended antarctic residence.

Psychoneuroendocrinology 26, 4: 421-431. http://dx.doi.org/10.1016/s0306-4530(00)00064-0

32. Lawrence A. Palinkas. 2001. Mental and cognitive performance in the cold. International Journal of Circumpolar Health 60, 3: 430-439.

33. Yong S. Park and Sung H. Han. 2010. Touch key design for one-handed thumb interaction with a mobile phone: Effects of touch key size and touch key location. International Journal of Industrial Ergonomics 40, 1: 68-76.

34. June Pilcher, Eric Nadler and Caroline Busch. 2010. Effects of hot and cold temperature exposure on performance: a meta-analytic review. Ergonomics. http://dx.doi.org/10.1080/00140130210158419

35. Richard L. Potter, Linda J. Weldon and Ben Shneiderman. 1988. Improving the accuracy of touch screens: an experimental evaluation of three strategies. In Proceedings of the SIGCHI conference on Human factors in computing systems, 27-32. http://dx.doi.org/10.1145/57167.57171

36. J. E. Reading, D. E. Roberts and W. K. Prusaczyk. 1997. Gender differences in finger temperatures during cold air exposure. San Diego, CA: Naval Health Reseach Center.

37. Enrico Rukzio, Karin Leichtenstern, Vic Callaghan, Paul Holleis, Albrecht Schmidt and Jeannette Chin. 2006. An Experimental Comparison of Physical Mobile Interaction Techniques: Touching, Pointing and Scanning. Springer Berlin Heidelberg. http://dx.doi.org/10.1007/11853565_6

38. Anthony J. Sargeant. Effect of muscle temperature on leg extension force and short-term power output in humans. European Journal of Applied Physiology and Occupational Physiology 56, 6: 693-698. http://dx.doi.org/10.1007/BF00424812
39. Andrew Sears, Mark Young and Jinjuan Feng. 2003. Physical disabilities and computing technologies: an analysis of impairments.

40. Paul R. Stang and Earl L. Wiener. 1970. Diver Performance in Cold Water. Human Factors: The Journal of the Human Factors and Ergonomics Society 12, 4: 391-399. http://dx.doi.org/10.1177/001872087001200405

41. Nancy J. Stone. 2003. Environmental view and color for a simulated telemarketing task. Journal of Environmental Psychology 23, 1: 63-78. http://dx.doi.org/10.1016/s0272-4944(02)00107-x

42. Warren H. Teichner. 1958. Reaction time in the cold. Journal of Applied Psychology 42, 1: 54. http://dx.doi.org/10.1037/h0049145

43. W. S. Vaughan. 1977. Distraction effect of cold water on performance of higher-order tasks. Undersea Biomedical Research.

44. J. A. Vaughan, E. A. Higgins, G. E. Funkhouser and E. M. Galerston. 1968. The effects of body thermal state on manual performance. AM-68-13. AM reports 68 , 13: 1-9.

45. W. S. Vaughan Jr and Michael B. Strauss. 1975. Exploratory Analysis of Predictors of Diver Performance Decrement During 3-Hour, Cold Water Exposures

46. Hannu Virokannas. Thermal responses to light, moderate and heavy daily outdoor work in cold weather. European Journal of Applied Physiology and Occupational Physiology 72, 5-6: 483-489. http://dx.doi.org/10.1007/BF00242279

47. Jacob O. Wobbrock. 2006. The future of mobile device research in HCI. In CHI 2006 workshop proceedings: what is the next generation of human-computer interaction, 131-134.

48. Jacob O. Wobbrock, Brad A. Myers and John A. Kembel. 2003. EdgeWrite: A Stylus-based Text Entry Method Designed for High Accuracy and Stability of Motion. In Proceedings of the 16th Annual ACM Symposium on User Interface Software and Technology, ACM, 61-70. http://doi.acm.org/10.1145/964696.964703

49. Mark A. Yeshnik. 1988. The variability of German winter temperature in relation to human performance and its implications for tactical military operations.

50. Yu Zhong, Astrid Weber, Casey Burkhardt, Phil Weaver and Jeffrey P. Bigham. 2015. Enhancing Android accessibility for users with hand tremor by reducing fine pointing and steady tapping. In Proceedings of the 12th Web for All Conference, 29. http://doi.acm.org/10.1145/2745555.2747277 